(C2008 IEEE. Personal use of this material is permitted. However, permission to reprint/republish this material for advertising or promotional purposes or for creating new collective works for resale or redistribution to servers or lists, or to reuse any copyrighted component of this work in other works must be obtained from the IEEE. 


\title{
Ontology based data warehouse modeling and managing ecology of human body for disease and drug prescription management
}

\author{
Shastri L. Nimmagadda ${ }^{1}$, Sashi K. Nimmagadda ${ }^{2}$ and Heinz Dreher ${ }^{3}$ Member, IEEE \\ ${ }^{1,2,3}$ Curtin Business School, Curtin University of Technology \\ (E-mail: shastri@eftel.com, sashi.nimmagadda@gmail.com,Heinz.Dreher@cbs.curtin.edu.au)
}

\begin{abstract}
Health care sector is currently experiencing a major crisis with information overload. With the increasing prevalence of chronic diseases and the ageing population the amount of paper-work is more than ever before. In the US, a hospital admission of one patient generates an estimate of 60 pieces of paper. The federal governments of various countries have passed policies and initiatives that focus on introducing information systems into the health care sector. Technology will immensely reduce the cost of managing patients and even reduce the risks of mis-diagnosing and prescribing incorrect medications to patients. This paper primarily focuses on introducing the concept of ontology based warehouse modelling and managing ecology of human body for disease and drug prescription management. Disorders of the human body and factors such as the patient's age, living and working conditions, familial and genetic influences can be simulated into Metadata in a warehousing environment. In this environment, various relationships are identified and described between these factors and the diseases. Secondly, we also introduce ontological representation of the various human body systems such as the digestive, musculoskeletal and nervous system in disease processes. Although this is an extensive and complex knowledge domain, the work in this paper is one of the first to attempt to introduce the use of ontology based data warehousing and data mining conceptually. We also aim at implementing and applying this research in practice.
\end{abstract}

Index Terms - data mining, data warehousing, disease management, ontology, human body, diseases

\section{INTRODUCTION}

The federal governments of various countries are taking the initiative to introduce information technology (IT) in the health care sector. Currently very few hospitals and health care providers are implementing IT in the delivery of their services. Nevertheless, IT is believed to potentially increase the quality, efficiency and safety of the health care sector. Due to these initiatives, several providers plan to increase their investments [27]. There are several types of technologies that the health sector is already using, including the electronic health record (EHR), computerised provider order entry (CPOE) and clinical decision support system (CDSS) [27]. They also mention that the spread of technology in the health care sector has been mainly in the administrative fields. Currently there is less diffusion of applications such as EHR, CPOE and CDSS.

A significant problem under discussion is the amount of paper-work that is increasing due to the increasing rates of chronic diseases and the rising number of hospital admis- sions. An executive research report indicated that in the US one patient admission generates an estimated 60 paper items [26]. This poses a challenge towards the environment as well as increases the costs.

Another challenging issue [20] faced by the medical industry is the management of patients who suffer from chronic illnesses such as diabetes, heart disease, asthma, and renal disease. For example, 100 million Americans [20] have at least one chronic condition, which is expected to rise to more than 150 million in the next 20-30 years. Furthermore, chronic conditions are the leading causes of death, disability and illness in US, accounting for more than $75 \%$ of direct medical expenditures. This creates challenges in how to handle increasing amounts of information in, both, clinical and research fields.

The aim of this paper is to provide a solution for the above problems through ontology based data warehouse modelling and data mining. The aim is also to investigate the feasibility of upgrading the EHR, CPOE, CDSS and interoperability with the modelling. Ontology based modelling is believed to form relationships between various entities and these entities can be well-connected with each other.

\section{LITERATURE SURVEY}

After an extensive literature search, in databases such as Elsevier and PubMed, we discovered that there are few good recent publications on the modelling under discussion. Few papers investigated the practical applications of ontology-based data warehousing with Metadata technology and data mining in the health sector.

Nevertheless, it was found through literature that the development of data warehouse provides easy access to accurate, relevant medical and business data [11]. This can enable health care professionals to make more informed decisions not only in patient diagnosis and management, but also in day-to-day operations and strategic planning [11].

Disease data warehouse $[1,36]$, facilitates the collection, storage and processing of specific data to facilitate numerous health care system applications, for example, electronic medical records, health insurance claim, medication, and lab systems. Entries from other databases can be extracted from external servers to integrate into the existing datasets.

The use of Metadata technology in decision support systems has recently started gaining popularity [33, 34, 35]. Little research has been done for its role in the health care sector. This paper discusses use of Metadata technology. 
Metadata technology which supports the warehousing environment is a product of integrated data, extracted from different sources. Specific patient queries, outcome analyses and reporting are performed from warehoused disease data. Hence, this will enable increased efficiency in storage and retrieval of patient information.

After a doctor interacts with a patient, the data gathered can be loaded daily into the data warehouse with periodic dimension. This capability is valuable when evaluating or discussing patients whose diseases have evolved over time or who have visited multiple physicians. Data patterns, correlations and trend studies can assist physicians in the diagnosis, management and prognostication of the patients. Very little research has been done in this area. However, the concept of ontology based molecular biology databases $[3,6,9]$ that has been derived based on several activities and functions of bio-genes, can be used to understand data patterns, trends and correlations in patient records. This will assist the doctor in diagnosing patient problems more effectively and promptly.

An independent database stores [10] sanitized data, retrievable from legacy, transaction oriented systems of display and uses formats, conducive to a specific purpose (research, outcome analysis). Transactional systems are poorly adapted to longitudinal or relational research. Relational models have not been efficient enough to support the high volumes and peak demands of transaction processing. An independent platform will interact with transaction systems, sending information in both directions. Many classes of users (physicians) performing both research and transaction oriented activities (e.g., examining patient's history, accessing outcome analysis, performing new treatments, tests, medications etc.) simultaneously monitor critical pathways. A set of applications that supplement current transaction systems to provide a repository based view of data stored in existing application files is needed.

MySQL and Oracle Relational DB managers [8] are used to design, develop and implement BioWarehouse. BioWarehouse integrates its component databases into a common representational framework within a single DBMS, thus enabling multiple database queries using SQL, besides facilitating comparative analysis and data mining.

An example of the use of data warehouse is [23], where the authors propose a comprehensive HIV prevention program evaluation data warehouse that can collect, manage, analyse and integrate large amounts of HIV prevention program data. This could reduce the costs associated with assessing HIV prevention efforts and could perform more sophisticated analysis and modelling. Historical HIV prevention data can be stored at the older detail level; recent data, at the current detail level. The data warehouse can generate brief or detailed summary reports. Data attributes (such as, year, severity of disease, progression and other variables), the algorithms used for summarization, and the mapping procedures from the operational environment, can also be stored in the data warehouse.

Several papers have been published recently regarding the use of ontology in practical applications by knowledge engineers. This is to address problems in the medical field such as ambiguity in terminology in the medical thesauri. To decrease the variability in the medical terminology and processing of pulmonary disease information by health care professionals, [25] have proposed to build several medical ontologies, specifically for the lung disease group. [24] have proposed ontologies for managing patients with heart failure. They emphasise that for the growing incidences of chronic diseases and increasing age, there needs to be an efficient way of establishing continuity in the care for patients. They believe that ontologies will act as 'glue' to ensure efficient work environment between health care professionals such as the doctors, nurses, physiotherapists and even the patients and their families.

Our paper introduces the idea of ontology based warehousing conceptually. Subsequent papers will focus on implementing this in practice - something that is likely to transform health care information management. Research is in progress; especially the development and implementation of our proposed data models. There are authors who are already working on similar concepts, but with different approaches [30], [32] and [25].

\section{STATEMENT OF PROBLEMS AND ISSUES}

Even if a large volume of medical information is available, pathological conditions are still poorly understood and diagnoses can be ambiguous [12]. Ontology based warehouse modelling is proposed with data mining procedures to extract knowledge on human body systems, from patterns of existing diseases. The data can be derived and conceptualized into ontologically derived data dimensions, based on these categories, functions and activities of human body systems. Simulation models can be deduced based on Metadata [16] and Data Warehousing [13,14] approaches.

An important aspect of human anatomy is learning the relationship (Fig. 1) between body structures and their location as interpreted through palpation of relevant anatomical landmarks. This examination eases the task of relating anatomical knowledge, observation and palpation to make or confirm diagnoses.

The conceptualization of data and information has a definite role in the logical and intelligent design of databases and data warehouses in which information is described as an inventory [18] and an asset to the organization. Two types of systems are interpreted: machine based (ontology perspective) and natural systems such as human anatomy. Integrated human anatomy [40] is a natural builtin system with well connected entities or dimensions. Dimensions analogous to entities can be described, conceptualizing the relationships, wherever necessary, among body systems such as skeletal, digestive, and muscular.

For example, relationships can be formed between the skeletal system, muscular system and the nervous system, as all three systems work in coordination with each other. This relationship results in the integrity of one system being dependent on the other. Several associated data dimensions are conceptualized using ontology. Volume of dimensions from real world situations can be interpreted through ontol- 
ogy conceptualization of the human anatomy and pathologies into the machine world. Study of various types of human structure, shape, colour and their patterns is useful in understanding the patient's history, diseases and type of drugs administered to these patients. Aging, environment and food habits affecting the human anatomy are also described to categorize likely related dimensions.

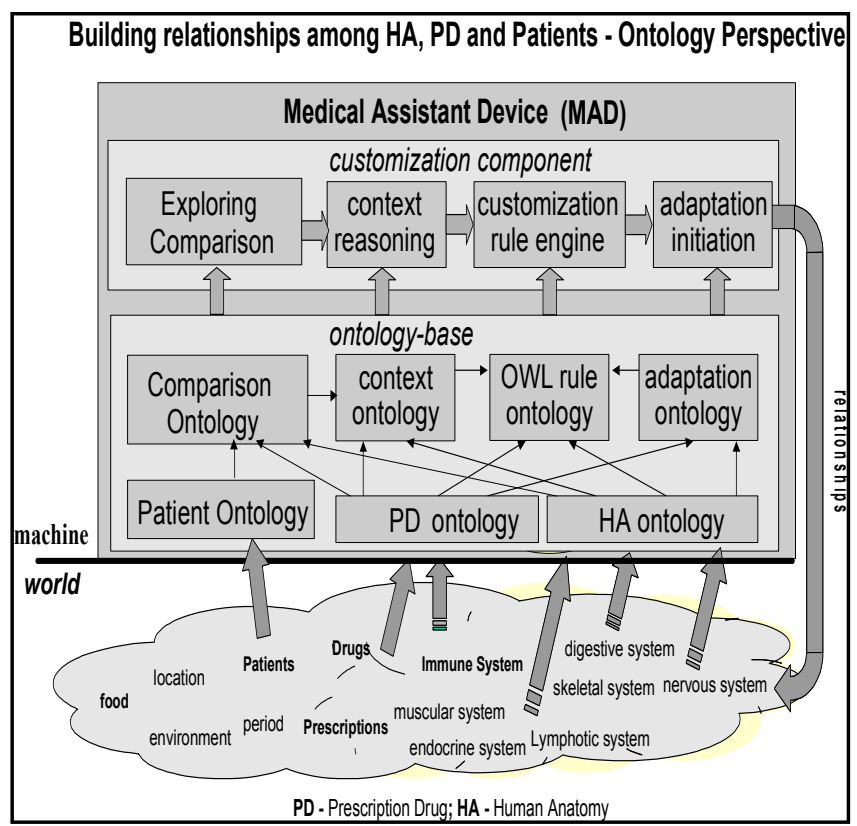

Fig.1. Interpreting real situations; building relationships using ontology

Different data gathered from several sources [17], can be conceptualized in terms of ontology, thus building relationships among related and un-related data dimensions through logical conceptualization of data dimensions.

Integration of data [3] from different sources and management of multiple data sources through Metadata and warehouse technologies are also some of the key issues. To ensure data quality and integrity, most large databases or data repositories adopt warehousing solutions and ETL (extract, transform and load) approaches. The proposed data warehousing is designed to integrate data from different sources across multiple platforms using a combination of scalable hardware and software systems to deliver accurate information across data sources and types.

Interoperability [5] has an immense scope and value in the expanding medical institutions, in which large population of patients are involved. Warehousing integrates its component databases into a common representational framework within a single DBMS, thus enabling multiple database queries using SQL, while facilitating comparative analysis and data mining.

\section{METHODOLOGY}

Two basic approaches, such as technological and methodological [15] are used to handle exponentially growing databases in many industries. As a matter of fact, technology offers solutions in storage capacity, processing power and access speed. Methodologies can derive indexing, data views, data mining and temporal databases. Basically, data warehouse is a concept of combined view of technology and methodology [15]. The objective is to curtail the size of database and warehouses without losing content and information value.

Before any data warehousing and data mining project is taken up, understanding medical data and the human body systems is important. Several methods are described in the literature for understanding human anatomy and physiology. The authors attempt to use ontology base [14] warehouse modelling [13] to extract knowledge of the human body systems and their systems (Fig. 2) interconnectivity.

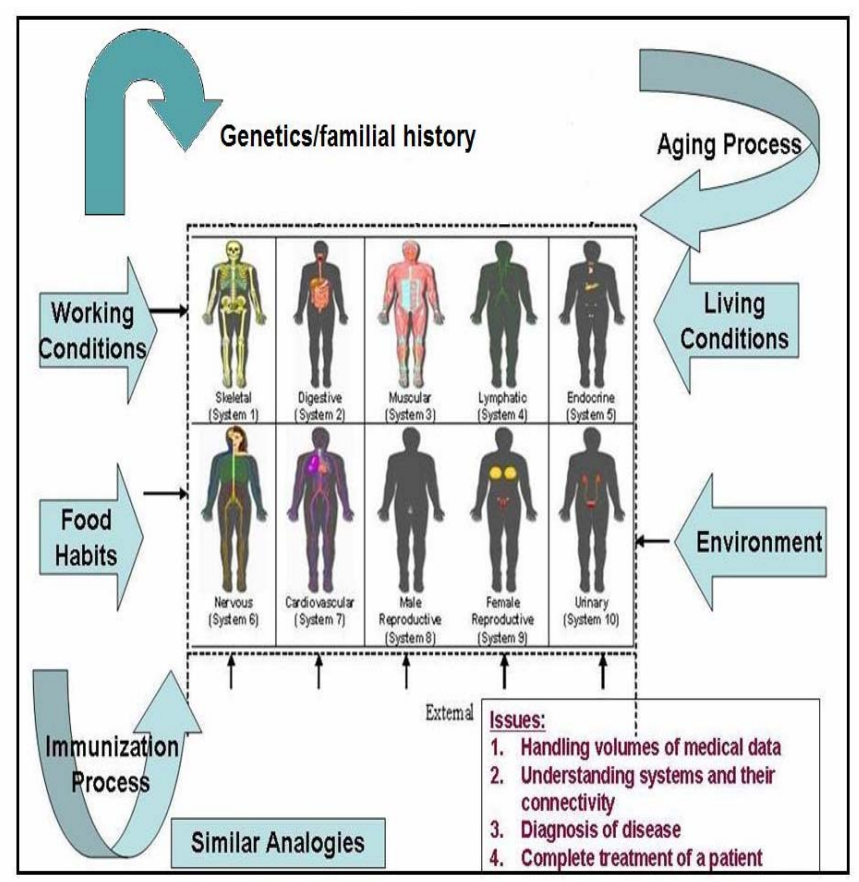

Fig.2. An integrated human anatomy model [39]

\section{DESCRIPTION OF DATA TYPES}

Like any databases, medical data structures are also heterogeneous. The data can be represented in hierarchical, relational and networking domains. All the historical data, based on age, disease, prescription, medication, period and geographic location are significant attributes of disease entity or dimension. These attributes can also be categorized as several dimensions, refining the multidimensional data structuring, which may enable data mining, ultimately enabling analysis of patient queries online. Details of patients, including health records and medication details must be made available for ascertaining the data integration process.

Data dimensions and attributes may be extracted from the historical data and conceptualized for the purpose of multidimensional data structuring and are discussed in the following sections. Data instances may be acquired for every attribute of each and every system's data dimension. The following is our attempt to structure four systems data dimensions as described in the following sections. 


\section{CONCEPTUALIZATION OF MULTIDIMENSIONAL DATA MODELS - ONTOLOGY PERSPECTIVE}

Several data entities of Human Anatomy are interpreted as dimensions in order to construct multidimensional data structures. Various factors affecting the human body (anatomy), as demonstrated in Fig. 3 are considered as several dimensions.

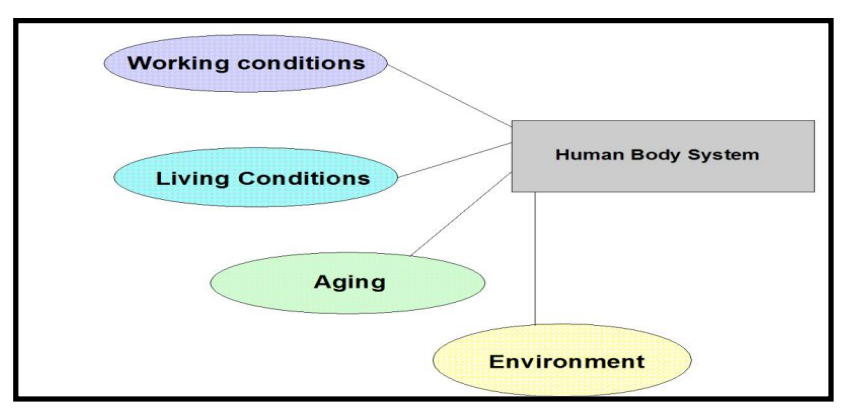

Fig.3. Human Body System - systems connectivity; factors affecting Human Anatomy

Multi-dimensional data structures are components for building the warehouse which integrates all the relationships based on ontology-conceptualization. Figs. 4 - 6 show ten major systems considered to integrate the dimensions and their associated attributes. Individual systems are described as major dimensions. Different data dimensions and their associated attributes have been derived and interpreted for structuring and represented in star-schemas. Please note that the diagrams are a snap-shot of a concept and, in no way fully describe the multi-dimensional ontology. Each system can be discussed in a comprehensive manner in subsequent papers.

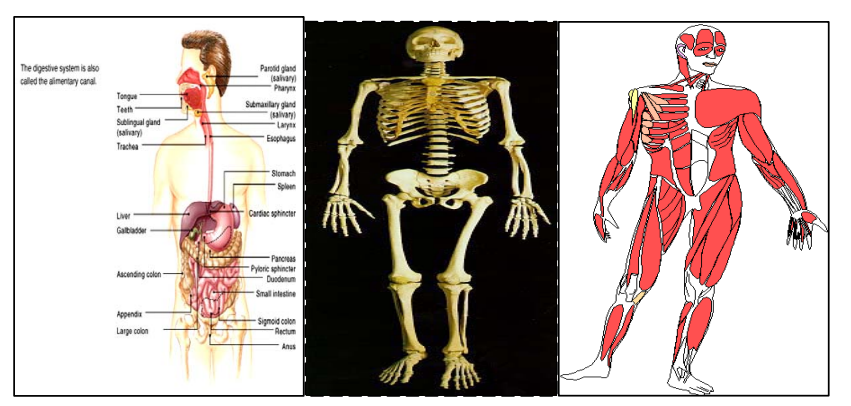

Fig.4. Figures showing entities and or dimensions, used in the data integration process

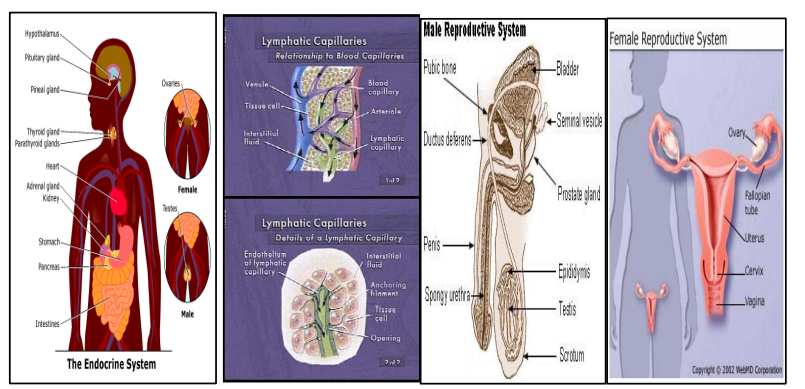

Fig.5. Figures showing entities and or dimensions, used in the data integration proce
Multiple data dimensions and attributes have been derived from skeletal and digestive systems (Figs. 7-9). These have been derived based on functions, hereditary, and other factors discussed earlier (Fig. 3). Based on data and how data patterns are represented for further data mining, multidimensional representation is logical and physical. Another form of dimensional representation of digestive system is modelled as shown in Fig. 8. Data attributes and their instances are denormalized for Fine grained structuring $[38,41]$. Decomposing all the dimensions will facilitate the data mining process. Similar multidimensional data structures for muscular, immune and nervous systems are characterized as shown in Figs. 10-12.

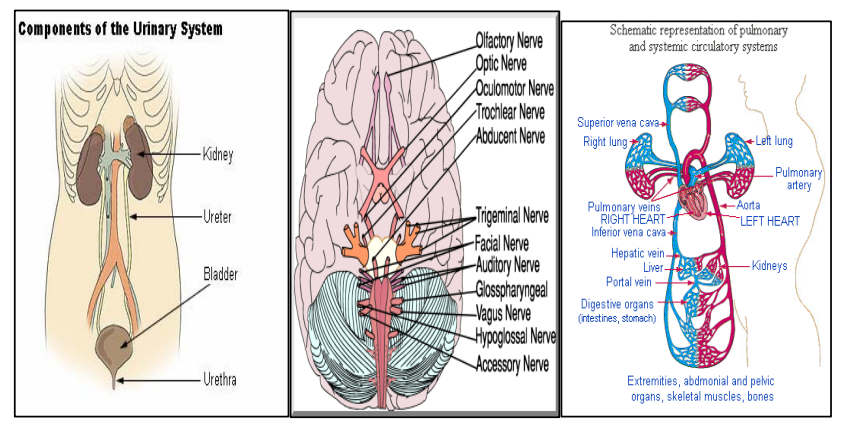

Fig.6. Entities and or dimensions, used in the data integration process

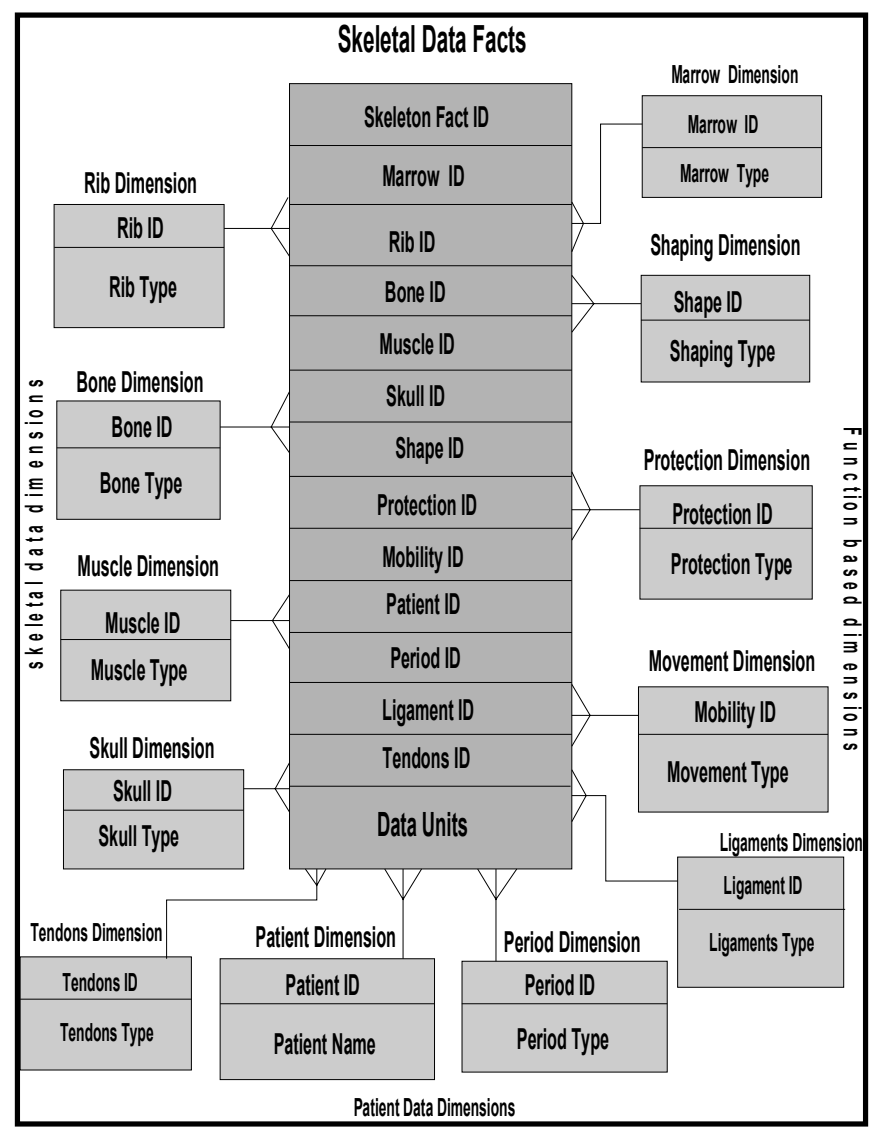

Fig.7a. Skeletal System Multidimensional Ontology - Data facts organization 
2008 Second IEEE International Conference on Digital Ecosystems and Technologies (IEEE DEST 2008)

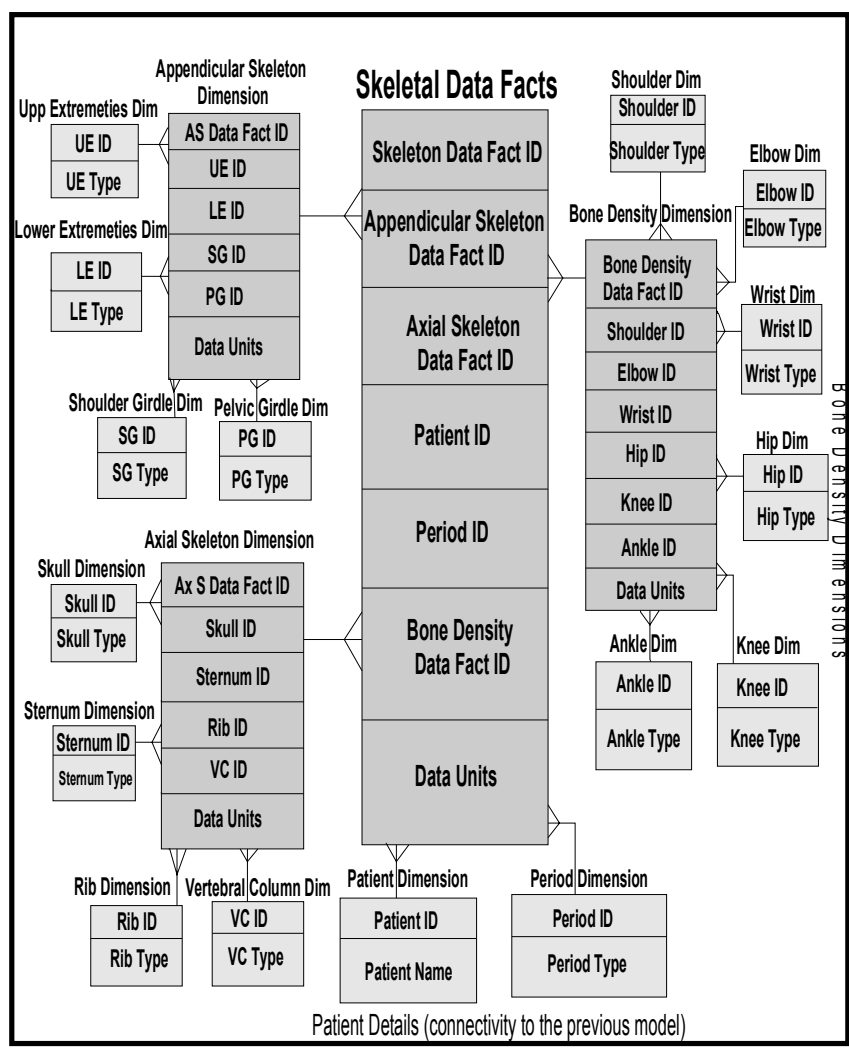

Fig.7b. Skeletal System Multidimensional Ontology - Data facts organization (based on functions)

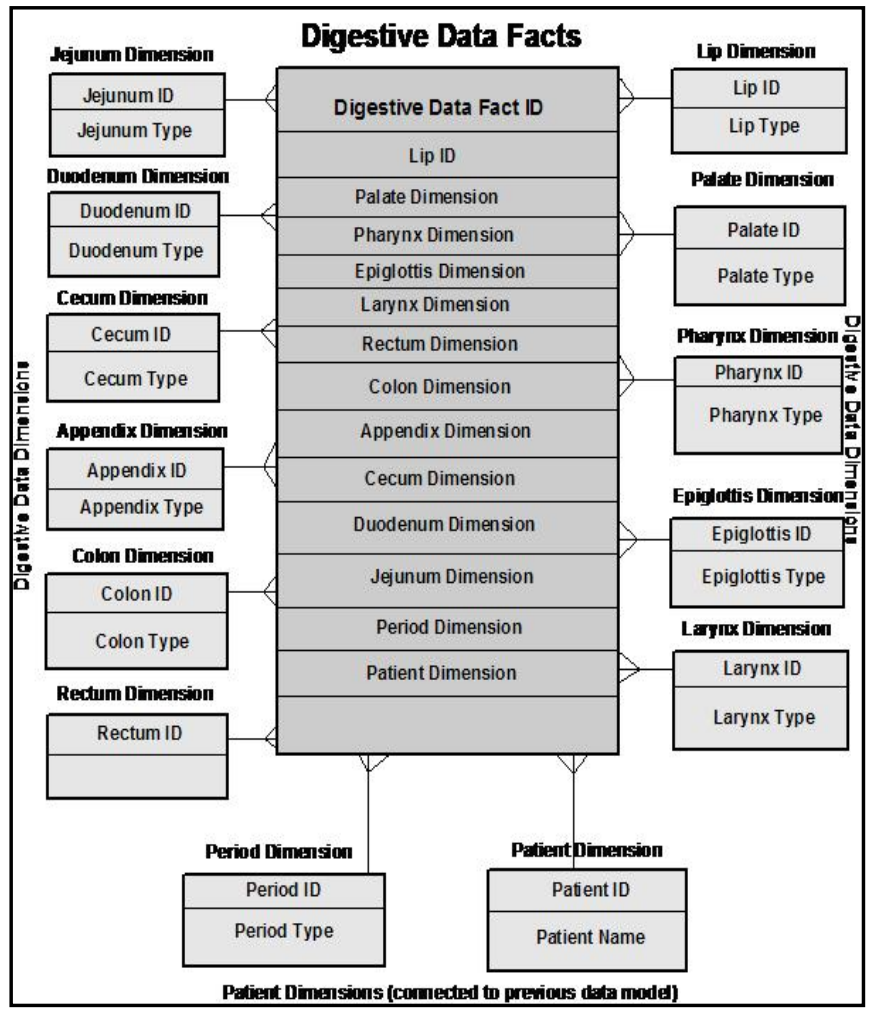

Fig.8. Digestive System Multidimensional Ontology - Data facts organization

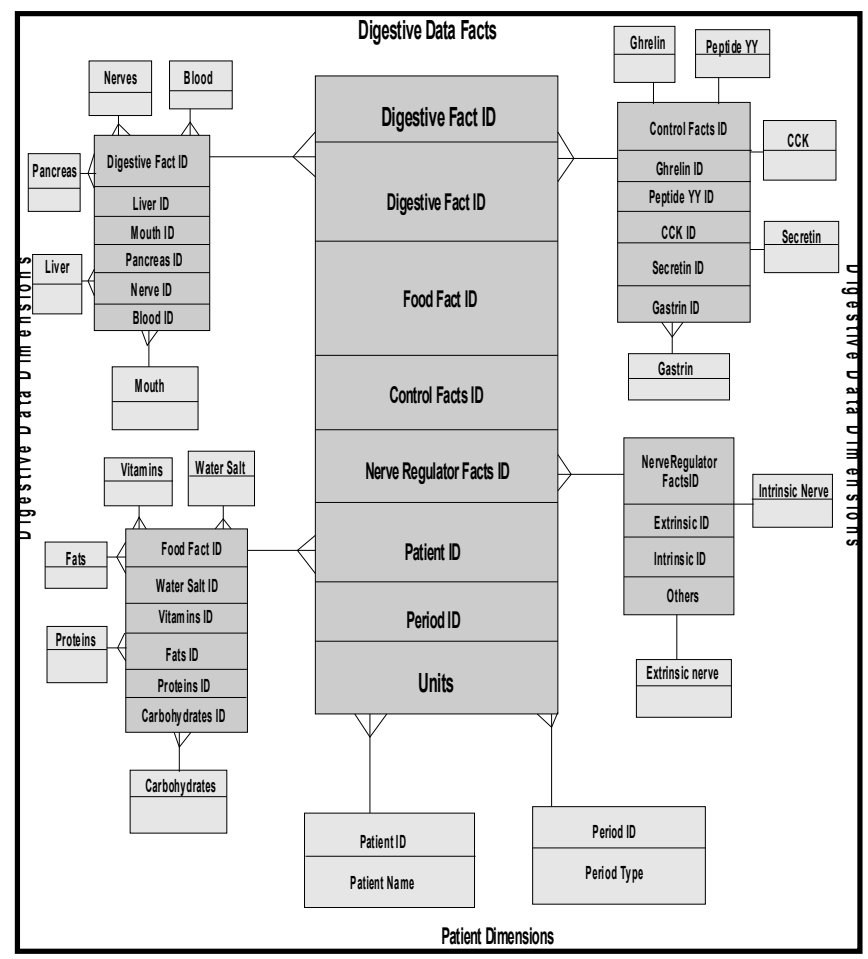

Fig.9. Digestive system ontology representation - separating data facts as per functions

Similar to skeletal and digestive data systems, data acquired from muscular system, are organized in a multidimensional representation as shown in Fig.10.

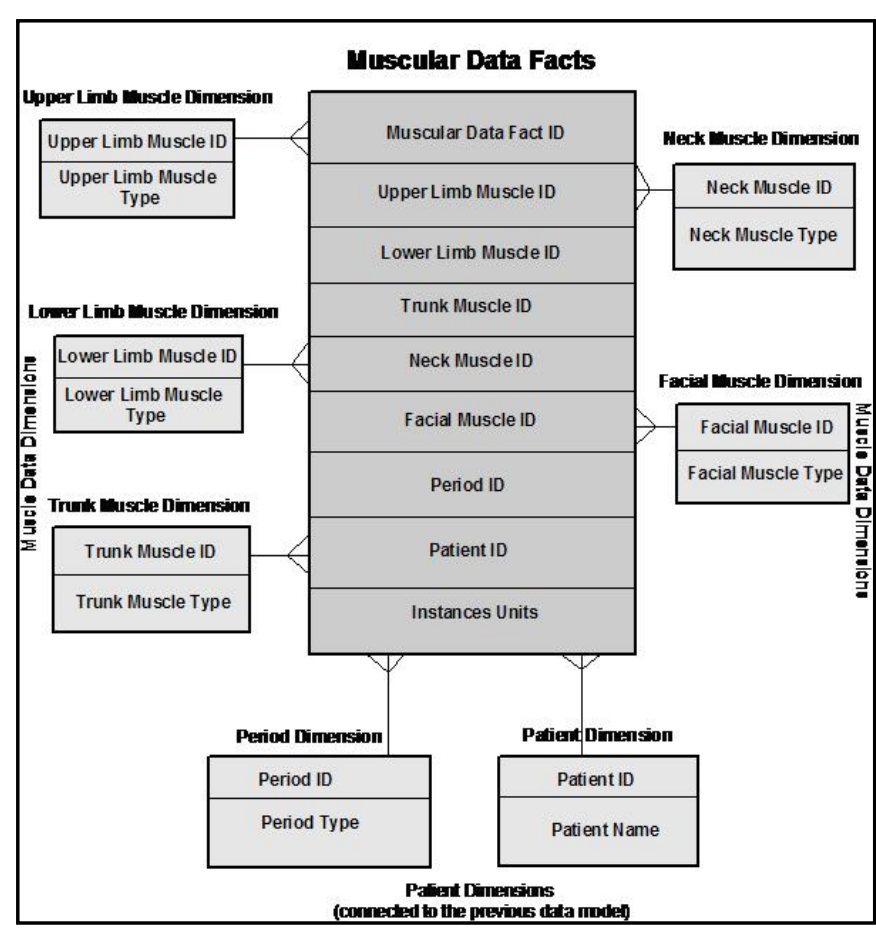

Fig.10a. Muscular System multidimensional ontology - data facts representation 
2008 Second IEEE International Conference on Digital Ecosystems and Technologies (IEEE DEST 2008)

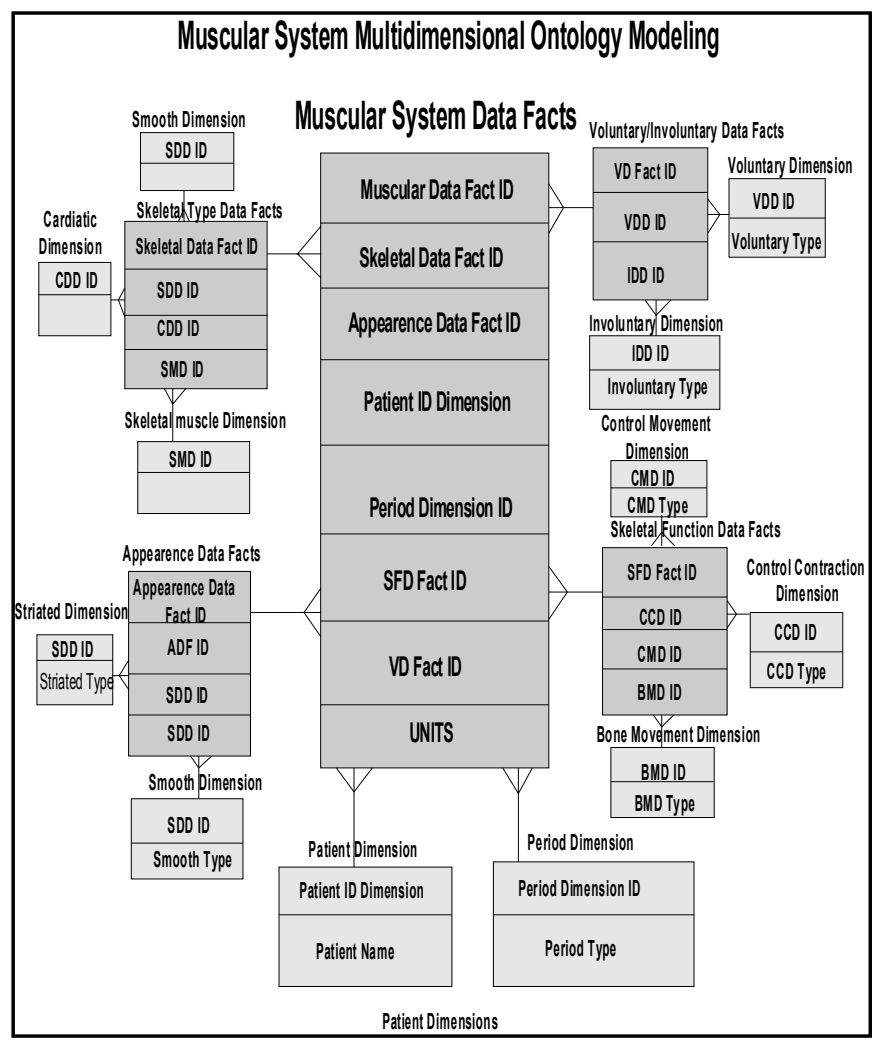

Fig.10b. Muscular system ontology representation - separating data facts as per functions

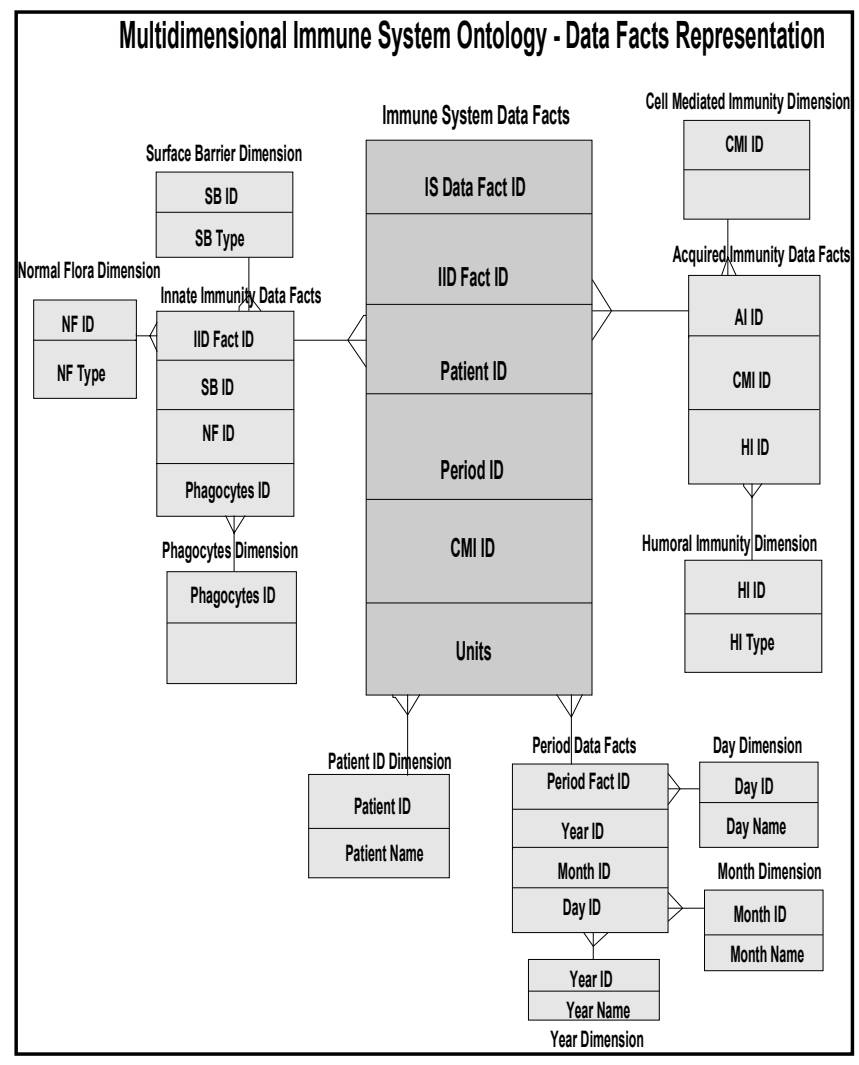

Fig.11. Immune System Multidimensional Ontology - Data Facts representation (snow-flake schema, with finer periodic data representation)

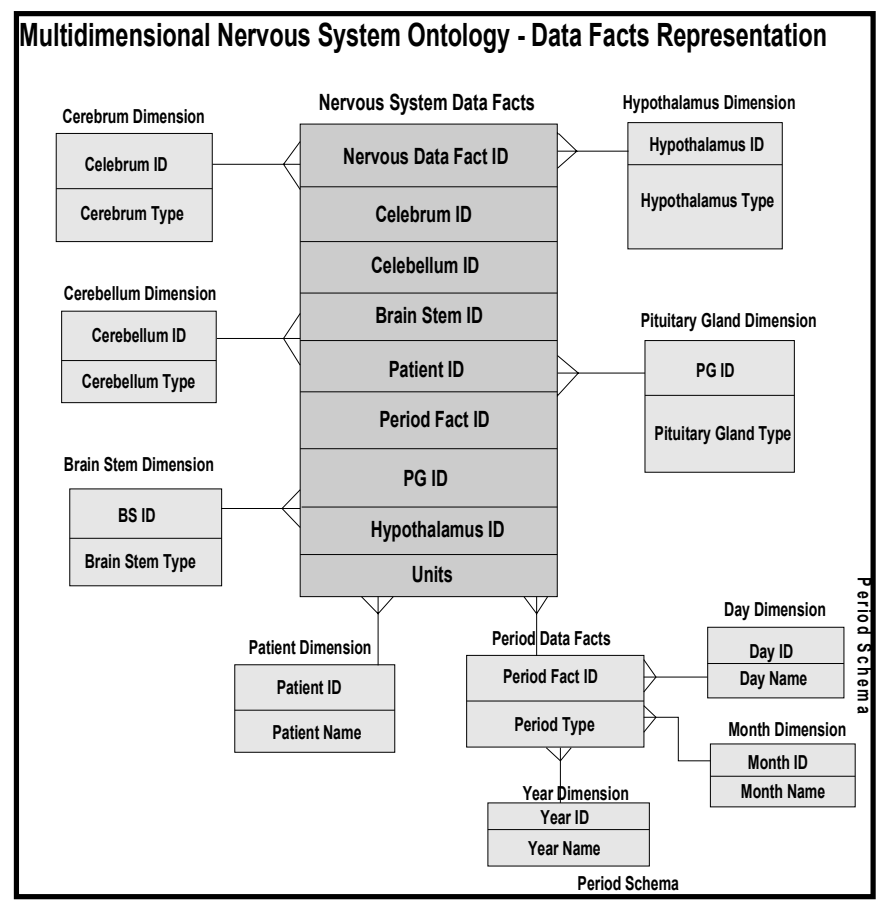

Fig.12. Nervous System Ontology - Data Fact Representation

\section{WAREHOUSING OF ONTOLOGY MODELS}

This is a data warehouse [1], with multidimensional metadata on human anatomy, describing the collection, storage and processing of specific data. This brings numerous health care system applications, electronic medical records, encounter, claim, medication and lab systems. Data from other databases can be extracted through external servers to integrate the existing datasets. Specific patient queries, outcome analysis and reporting, are performed from warehoused disease data.

Multi-dimensional data structuring and data integration are key components in a warehouse environment. Metadata is an intelligent logical data storage, which is at the core of warehoused medical data. Ten major systems described in the previous section (Figs. 4-6) are interpreted as major data dimensions in which all inherent dimensions are categorized and re-interpreted for building relationships. All these major dimensions are well interconnected and we must ensure that there is no ambiguity in narrating the relationships among these dimensions, especially addressing semantics and taxonomy. A warehouse framework is proposed (Fig. 13) containing data acquisition, ontologically structured multiple medical data dimensions, and integrated in a warehouse environment and finally mining different data views for data interpretation. As shown in Fig. 13, all the entities/dimensions from body systems are ontologically conceptualized, building relationships. These conceptualized data dimensions are interconnected in a warehousing environment in the form of Metadata structure. 
2008 Second IEEE International Conference on Digital Ecosystems and Technologies (IEEE DEST 2008)

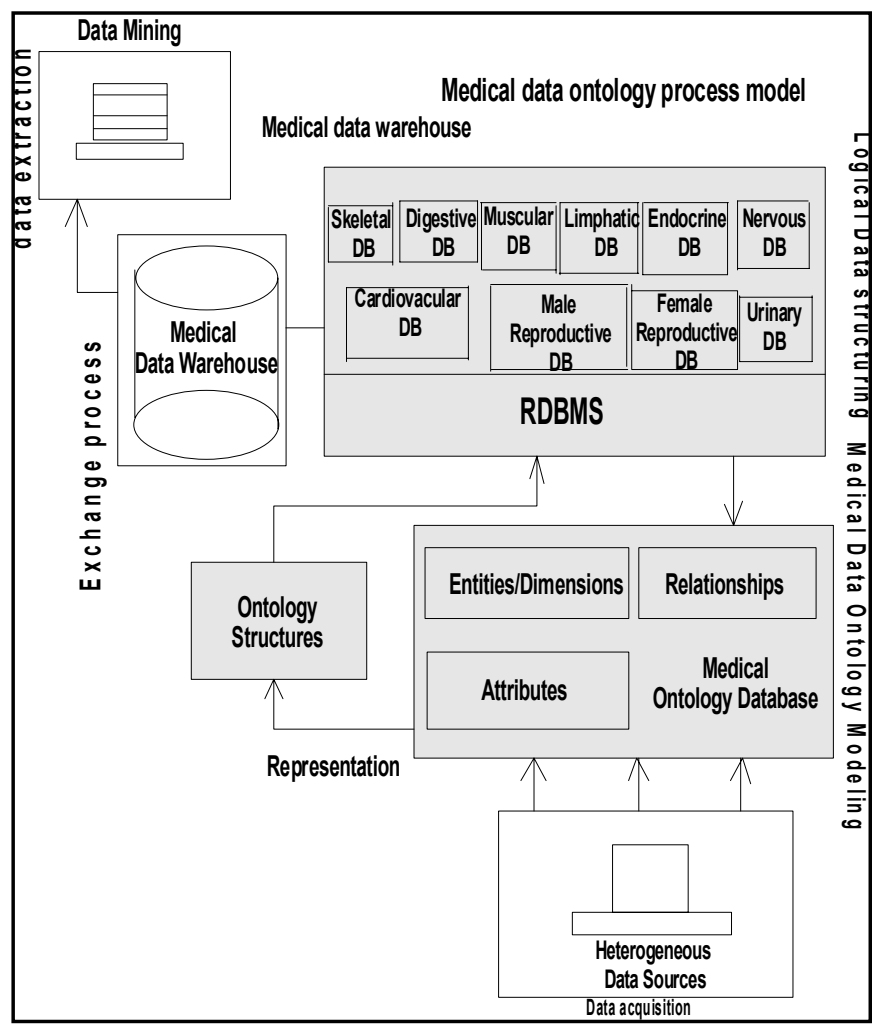

Fig.13. Data Warehouse Framework - Metadata Process Model

All these ontologically derived structures are integrated (Fig. 14) to accomplish Metadata in a warehouse. It is important for data warehouse designers to define the scope, depth, comparability and accuracy of data being entered into the warehouse.

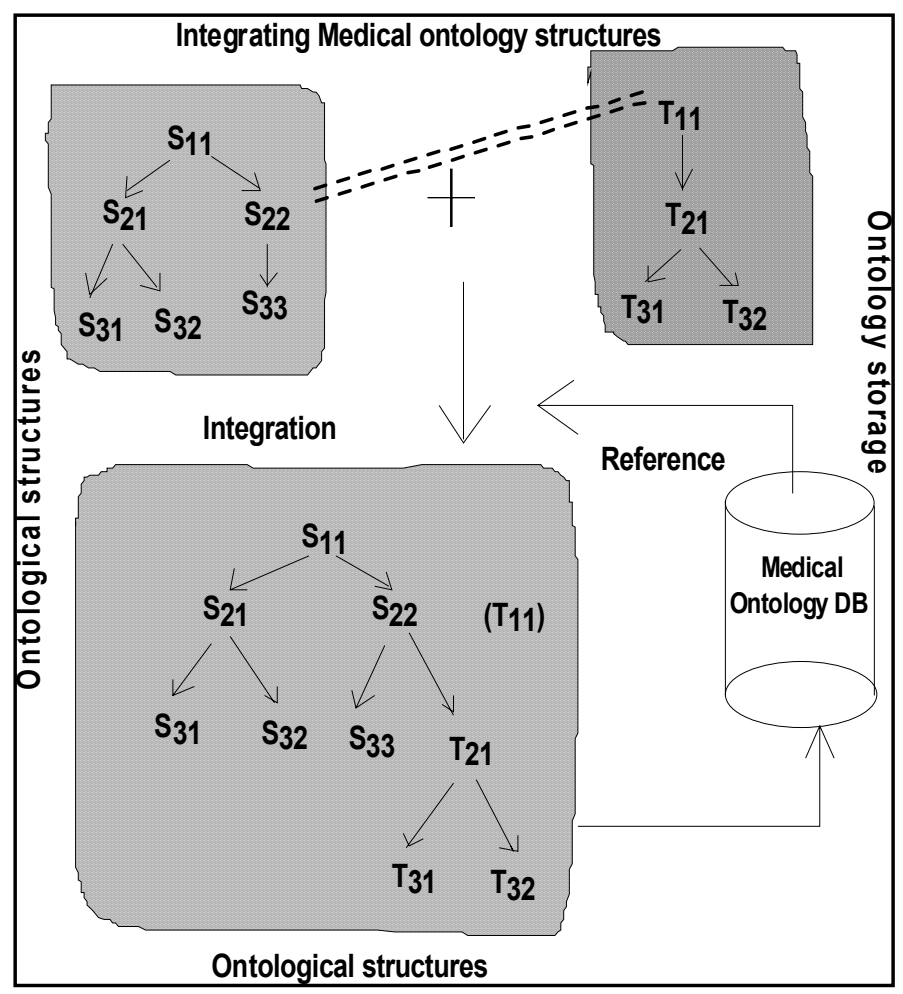

Fig.14. Data Integration - Ontology Structures

\section{ISSUES OF IMPLEMENTATION}

Data mining of warehoused medical data is performed from the existing mining algorithms. These algorithms support many decision support systems in the market. Decision support systems application assists providers in treating the patients, where electronic patient data are selected and analysed to generate comments and notes for the patient records. These types of decision support systems can support both primary care provider and clinician. These systems ensure the consistency of messaging and continuity of care that may be essential for a large population of patients.

The effective use of massive volumes of patient and other medical data is an issue for all enterprises. Ontology based data warehouse, once designed and developed, the medical practitioner can now mine the warehouse via patient queries and analyse results to make decisions. Capturing, cleaning, storing and managing data for decision support can generate a range of reporting applications through ad hoc query capabilities, visualization and drill-down functions via OLAP (On Line Analytical Processing) tools [21]. The OLAP engine provides an interface for querying summarized and aggregate information across multiple dimension-hierarchies. Data mining supports automatic exploration of these datasets. It attempts to source out patterns and trends in the data and infer rules from these patterns.

Implementing enterprise wide databases in the medical industry is a major challenge [4], due to the increasing amount of information, most of which is fragmented. Diseases can be managed through precise understanding of the inter-connectivity among human body systems and overall knowledge of human anatomy, physiology and pathologies. Warehoused data can be used for mining different data views, captured from different disciplines and patient queries. Metadata (such as years, severity, progression of diseases and other variables), the algorithms used for summarization, and the mapping from the operational environment to the data warehouse, can also be stored in the data warehouse.

An example is in the clinical laboratory immunology [11] field. Physicians can load data every day into the data warehouse with periodic dimension. This capability is valuable when evaluating and discussing patients whose disease has evolved over time or who visited multiple physicians. Data patterns, correlation and trend studies assist physicians in the diagnosis, management and prognostication of the patient. An independent platform will interact with transaction systems sending information in both directions. There can be many classes of users (bi-lateral), (physicians) performing both research and transaction oriented activities (e.g., examining patient's history, accessing outcome analysis, performing new treatments, tests, medications etc.), while simultaneously monitoring critical path ways. Conclusions and recommendations made, though are inclusive, but are useful for medical professionals and practitioners involved in managing drug prescriptions and patients data records. 


\section{CONCLUSIONS AND RECOMMENDATIONS}

When the data are integrated, a patient's risk, treatment and health status can be better determined. Data warehousing offers the health care industry an opportunity to accumulate and assimilate information from numerous data feeds (e.g., laboratory, pharmacy, radiology, finance, etc.) and make it available for decision-making. Data warehousing offers the health care industry an opportunity to accumulate and assimilate information from numerous data feeds (e.g., laboratory, pharmacy, radiology and finance) and make it available for decision-making. Hence, EHR, CPOE and CDSS can be upgraded towards more efficiency. The goals of a data warehouse are: (a) to make an organization's information accessible; (b) to make the organization's information consistent; (c) to be an adaptive, reliable, and resilient source of information; (d) to be a secure bastion that protects the information assets; and (e) to be the foundation for decision-making. Our future research will focus on the employment of modelling in real-life practical situations.

Efficient management, in the $21^{\text {st }}$ century, of clients, data, personnel, costs and time can be challenging. Development and implementation of a lab data warehouse that is a dimensionalized relational data-base with variety of multi-datamarts requires the OLAP tool for analysis. The data warehouse can provide easy access to accurate and relevant medical and business data that can be provided to assist clinicians to make more informed decisions. Beyond the scope of clinical laboratory immunology, the data warehouse offers a powerful tool in acquiring factual data to assist in the revamping of healthcare. Traditional institutional boundaries must be replaced with cooperation, collaboration and the sharing of scarce resources to focus on providing high-quality care to patients. The data warehouse may offer a solution to derive and to present the factual information that will assist in the redesign of the health care system so as to enable quality healthcare. The implementation of a data warehouse requires commitment, readiness in usage and a willingness to evolve through the information management restructuring process.

\section{Acknowledgement}

Authors acknowledge reviewers critical comments and suggestions. Prof Elizabeth Chang is especially thanked for useful discussions.

\section{REFERENCES}

[1] Anonymous, (1998) Metadata releases disease data warehouse for outcomes analysis and reporting, Health Care Strategic Management; 16, 4; Health \& Medical Complete, Health Care Strategic Management

[2] Anonymous. (2000) Merging lab, drug data boosts disease management. Data Strategy Benchmarks, February: 28-32.

[3] Davidson, S.B, Overton C, Buneman P. (1995) Challenges in integrating biological data sources. Journal of Computational Biology, 2(4):557-572.

[4] Geisler, M.A, Will D. (1998) Implementing enterprise wide databases: a challenge that can be overcome. Top Health Info Manage, 19:118 .

[5] Karp, P. (1995) A strategy for database interoperation. Journal of Computational Biology, 2(4):573-586.
[6] Karp, P.D. (2000) An ontology for biological function based on molecular interactions. Bioinformatics, 16(3):269-285.

[7] Kuperman, G. J., Aurelia B., Curt C., Bruce F., Stetson, P. D., Cooper, M. (2006) Using IT to Improve Quality at New York - Presbyterian Hospital: A Requirements-Driven Strategic Planning Process, AMIA 2006 Symposium Proceedings Page - 449

[8] Lee, T.J, Pouliot, Y.,Wagner, V. Gupta, P., Stringer-Calvert, D.W.J., Tenenbaum, J. D., and Karp, P.D, (2006) BioWarehouse: a bioinformatics database warehouse toolkit BMC Bioinformatics2006, 7:170 doi:10.1186/1471-2105-7-170.

[9] Markowitz, V. (1995) Heterogeneous molecular biology databases. Journal of Computational Biology, 2(4):537-538.

[10] Mathys, G. R, (1995) Patient-care systems trends: The clinical data repository, Health Management Technology; 16, 2; Health \& Medical Complete, pg. 12

[11] Miller, G.C, Dolan, C.T, Crowson, N., Stout, S.R. (2002) Data warehousing and information management strategies in the clinical immunology laboratory, Clinical and Applied Immunology Reviews 3, 73-86, 2002 Elsevier Science Inc.

[12] Cotran R and Robbins S (1999): Pathologic Basis of Disease. (1st ed.) Elsevier Health Sciences, New York.

[13] Nimmagadda, S.L. and Dreher, H. (2006) Ontology-Base Data warehousing and Mining Approaches in Petroleum Industries: in Negro, H.O., Cisaro, S.G., and Xodo, D., (Eds.), Data Mining with Ontologies: Implementation, Findings and Framework, a book published in 2007 by Idea Group Inc. http://www.exa.unicen.edu.au/dmontolo/

[14] Nimmagadda, S.L. and Dreher, H. (2005) Ontology of Western Australian petroleum exploration data for effective data warehouse design and data mining, a paper presented and published in the proceedings of the $3 \mathrm{rd}$ international IEEE conference on Industrial Informatics, held in Perth, Australia, August, 2005.

[15] Orly, K., Boaz, R., Israel, P. (2003) A selective Data retention approach in massive databases, Omega, The International Journal of Management Science, 32, 87-95p.

[16] Nimmagadda, S. L. and Dreher, H (2007) DESIGN OF PETROLEUM COMPANY'S METADATA AND AN EFFECTIVE KNOWLEDGE MAPPING METHODOLOGY, a paper accepted for oral presentation in the IASTED conference to be held in Cambridge in USA, November 2007.

[17] Nimmagadda, S.L. and Rudra, A. (2004d) Data sources and requirement analysis for multidimensional database modelling - an Australian Resources Industry scenario, published in the proceedings in the 7th international conference on IT, held in Hyderabad, India.

[18] Ronen, B, Spiegler, I. (1991), Information as inventory: a new conceptual view, Information \& Management; 21: 239-47p.

[19] Rudra, A. and Nimmagadda, S.L (2005) Roles of multidimensionality and granularity in data mining of warehoused Australian resources data, Proceedings of the 38th Hawaii International Conference on Information System Sciences, Hawaii, USA

[20] Perry, T. (2004) Technology-Driven Outcomes Health Management Technology; 25, 1; Health \& Medical Complete pg. 40

[21] Pujari, A.K. (2002). Data Mining Techniques, Universities Press (India) Private Limited, p. 7-67

[22] Saladin K (2001) Anatomy and Physiology - The unit of form and function. (2nd ed.) New York: McGraw-Hill

[23] Xiaohong, M. D., Choi K W., Levi R., Xiao-Jun W, Bertram T. II, (2002) A data warehouse concept for HIV prevention program evaluation, AIDS Education and Prevention; 14, 3; Health \& Medical Complete, pg. 120.

[24] Eccher C, Purin B, Pisanelli DM, Battaglia M, Apolloni I and Forti $\mathrm{S}$ (2006) Ontologies supporting continuity of care: The case of heart failure. Computers in Biology and Medicine 789-801.

[25] Baneyx A, Charlet J and Jaulent M (2007) Building an ontology of pulmonary diseases with natural language processing tools using textual corpora. International Journal of Medical Informatics 76: 208-215

[26] Health Information and Management Systems Society (2003): Executive Research Report - Paper crisis in American Company. Lanier Worldwide, Inc. - A Ricoh Company (United States)

[27] Medpac (2004): Information Technology in Health Care. Chapter 7, Report to the Congress: New Approaches in Medicare - Washington DC.

[28] Department of Communications, information technology and the arts (2004): Executive report - Australia's strategic framework for the information economy 2004-2006; Opportunities and challenges for the Information age. 
[29] Thomson TG (2004): The decade of health information technology: Delivering Consumer-centric and Information rich health care. Framework for Strategic Action 2004. Washington DC.

[31] Szirbik, N.B., Pelletier C., and Chaussalet T (2006): Six methodological steps to build medical data warehouses for research. International Journal of Medical Informatics V - 75 p. 683-691.

[32] Documentation available at openehr.org

[33] Sen, A. (2004) Metadata management: past, present and future. Decision Support Systems 37: 151-173.

[34] Lawrence A, W Jr and Hess T.J. (2002) Metadata as a knowledge management tool: supporting intelligent agent and end user access to spatial data. Decision Support Systems 32: 247-264.

[35] Ahmad I, Azhar S and Lukauskis P (2004): Development of a decision support system using data warehousing to assist builders/developers in site selection. Automation in Construction 13: 525 -542.
[36] Ramick, D.C (2001): Data Warehousing in Disease Management Programs. Journal of Healthcare information management 15: 99-105

[37] Tremblay MC, Fuller R, Berndt D and Studnicki J (2007): Doing more with more information: Changing healthcare planning with OLAP tools. Decision Support Systems. 43: 1305-1320

[38] Shin SK and Sanders GL (2006): Denormalization strategies for data retrieval from data warehouses. Decision Support Systems. 42: 267-282.

[39] Figure designed from a model at http://www.innerbody.com/htm/body.html

[40] Moore K. L and Dalley A. F (1999). Clinically Orientated Anatomy. (4th ed.) Pennsylvania: Lippincott Williams and Wilkins.

[41] Hoffer J A, Prescott M and McFadden F (2001): Modern Database Management. (6th ed.) New Jersey: Prentice Hall. 